\title{
Symmetry breaking and criticality in tensor-product states
}

\author{
Chen Liu, ${ }^{1}$ Ling Wang, ${ }^{2}$ Anders W. Sandvik, ${ }^{1}$ Yu-Cheng $S u,{ }^{3}$ and Ying-Jer Kao ${ }^{3}$ \\ ${ }^{1}$ Department of Physics, Boston University, 590 Commonwealth Avenue, Boston, Massachusetts 02215, USA \\ ${ }^{2}$ Institut der Theoretischen Physik, Universität Wien, Boltzmanngasse 3, A-1090 Vienna, Austria \\ ${ }^{3}$ Department of Physics and Center of Quantum Science and Engineering, National Taiwan University, Taipei, Taiwan
}

(Dated: October 30, 2018)

\begin{abstract}
We discuss variationally optimized matrix-product states for the transverse-field Ising chain, using $D \times D$ matrices with small $D \in\{2-10\}$. For finite system size $N$ there are energy minimums for symmetric as well as symmetry-broken states, which cross each other at a field value $h_{c}(N, D)$; thus the transition is first-order. A continuous transition develops as $N \rightarrow \infty$. The asymptotic critical behavior is then always of mean-field type (the magnetization exponent $\beta=1 / 2$ ), but a window of field strengths where true Ising scaling holds $(\beta=1 / 8)$ emerges with increasing $D$. We also demonstrate asymptotic mean-field behavior for infinite-size two-dimensional tensor-product (iPEPS) states with small tensors.
\end{abstract}

PACS numbers: 75.10.Jm, 75.40.Mg, 75.40.Cx, 05.30.Rt

Methods based on matrix-product states (MPSs) [1, 2] have become the primary computational tools for studies of static as well as dynamic properties of one-dimensional quantum many-body systems [3]. Key steps in the development of these methods were White's density matrix renormalization group (DMRG) [4, 5], the demonstration by Östlund and Romer of its connection to MPSs [2], and later important insights from the field of quantum information theory. In particular, the concept of entanglement entropy (the area law) both explains the success of the approach in one dimension and its failure (violation of the area law) in higher dimensions [6 8]. The formulation of computational methods directly in terms of MPSs also led to a framework for efficient optimization of these states independently of the DMRG method 9 11, and to a long-sought way of computing time evolution [12]. The MPS approach also has a natural extension to higher dimensions which does obey the area law 7] - tensor-product states; also referred to as projected-entangled-pair-states (PEPSs) [13, 14].

In spite of numerous successful applications of MPSbased methods, some fundamental aspects of this class of quantum states have not yet been studied in detail. It is well known that the finite size $D$ of the $D \times D$ matrices (the elements of which are the variational parameters) imposes a finite correlation length, and recently it has been recognized that scaling in $D$ for infinite system size $N$ can be carried out as an alternative to finite-size scaling 15] (i.e., $D$ and $N$ can be considered as different but equally valid ways to regularize the calculations). As in mean-field theory (which corresponds to $D=1$ ), an MPS can break symmetries of the hamiltonian at a phase transition. Exactly how the critical behavior of the order parameter (the true scaling exponent $\beta$ ) emerges as a function of $N$ and $D$ has not been studied systematically, however. This may be partially due to technical challenges in properly optimizing an MPS close to a phase transition. Such issues are present also for the PEPS ap- proach in two dimensions. Order-parameter curves often exhibit rounding [17], that may appear due to incomplete convergence, approximations made 15, or due to external fields included to stabilize the calculation [18]. Nevertheless, the behavior slightly away from the transition can be well described by the expected critical exponent [15, 16, 19]. The question remains whether this is the true critical behavior of the MPS or PEPS variational ansatz with finite $D$, or whether there could eventually be a cross-over to a different asymptotic form.

In this Letter we study the asymptotic critical behavior by using numerically stable high-precision optimization methods for small $D$, for both finite and infinite $N$. Using the transverse-field Ising model as a demonstration, we show that access to the true critical behavior of an MPS requires very high numerical precision; in some cases higher than the double-precision (64-bit) floating point arithmetic normally used. Optimizing the states to the required precision, we show that the asymptotic critical behavior of the order parameter is always meanfield like $(\beta=1 / 2)$. The true universal exponent for the model $(\beta=1 / 8)$ emerges in a window which approaches the critical point as $D$ increases. We also shows results in two dimensions for an infinite-size PEPS (iPEPS), optimized using a recently developed numerically stable scheme [20]. Also here we find $\beta=1 / 2$ asymptotically.

First, consider the simplest kind of MPS for a periodic, translationally invariant $S=1 / 2$ spin chain;

$$
|\Psi\rangle=\sum_{\left\{\sigma^{z}\right\}} \operatorname{Tr}\left\{A\left(\sigma_{1}^{z}\right) A\left(\sigma_{2}^{z}\right) \cdots A\left(\sigma_{N}^{z}\right)\right\}\left|\sigma_{1}^{z}, \ldots, \sigma_{N}^{z}\right\rangle,
$$

where $\sigma_{i}^{z}= \pm 1$ and $A( \pm 1)$ are two hermitian $D \times D$ matrices. As illustrated in Fig. 1, the normalization of this state can be expressed as the contraction of a network of 3-index tensors $A_{a b}(\sigma)$, where $\sigma= \pm 1$ is the physical index. By contracting over the physical indices first, matrices $B$ of size $D^{2} \times D^{2}$ are obtained;

$$
B_{i j}=A_{a b}(+1) A_{c d}^{*}(+1)+A_{a b}(-1) A_{c d}^{*}(-1),
$$


(a)

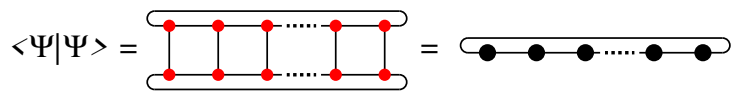

(b)

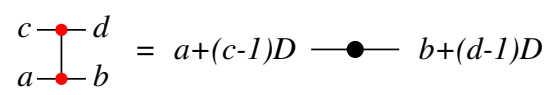

FIG. 1: (Color online) (a) The norm of an MPS expressed as the contraction of a tensor network. Carrying out the summations over the spin indices first (vertical bonds), as indicated in (b), gives a simple trace of a product of matrices of size $D^{2} \times D^{2}$ (with a possible labeling of the elements indicated).

where $i=a+(c-1) D$ and $j=b+(d-1) D$. The normalization is then simply

$$
\langle\Psi \mid \Psi\rangle=\operatorname{Tr}\left\{B^{N}\right\} .
$$

Expectation values can be computed in a very similar way, with some of the $B$ matrices in the product replaced by the matrix obtained as in (2) but with the operator in question first acting on the physical index [3]. For instance, the magnetization $m$ is given by

$$
m=\left\langle\sigma_{i}^{z}\right\rangle=\frac{\operatorname{Tr}\left\{M B^{N-1}\right\}}{\operatorname{Tr}\left\{B^{N}\right\}}
$$

where the matrix $M$ is

$$
M_{i j}=A_{a b}(+1) A_{c d}^{*}(+1)-A_{a b}(-1) A_{c d}^{*}(-1) .
$$

The generalization to expectation values of products of two or more operators is straight-forward.

The matrix $B$ is exactly analogous to the transfer matrix in classical statistical mechanics. With $U$ the unitary matrix that diagonalizes $B$, giving its eigenvalues $\lambda_{1}, \ldots, \lambda_{D^{2}}$, the magnetization can be written as

$$
m=\frac{\sum_{i}\left[U^{-1} M U\right]_{i i} \lambda_{i}^{N-1}}{\sum_{i} \lambda_{i}^{N}} .
$$

As in the transfer-matrix approach, the $N \rightarrow \infty$ limit can be taken by keeping only the leading eigenvalue; assumed here to be $\lambda_{1}$. The magnetization is then

$$
m=\frac{1}{\lambda_{1}} \sum_{i, j} v_{1 i}^{*} v_{1 j} M_{i j},
$$

where $v_{1}$ is the eigenvector of $B$ corresponding to $\lambda_{1}$.

Given a hamiltonian $H$, the problem is how to find the matrices $A( \pm 1)$, of given size $D$, that best reproduce the ground state. This can be formulated as a variational problem; to minimize the energy $E=\langle\Psi|H| \Psi\rangle$. Several different optimization methods have been developed. For finite $N$, the translational invariance is typically broken as a series of local optimizations are carried out, sweeping back and forth through an open chain [3] (similar to DMRG calculations [4, 5]). In a periodic chain, where the calculation is more demanding, uniformity is gradually restored as the matrices converge. For $N=\infty$, the
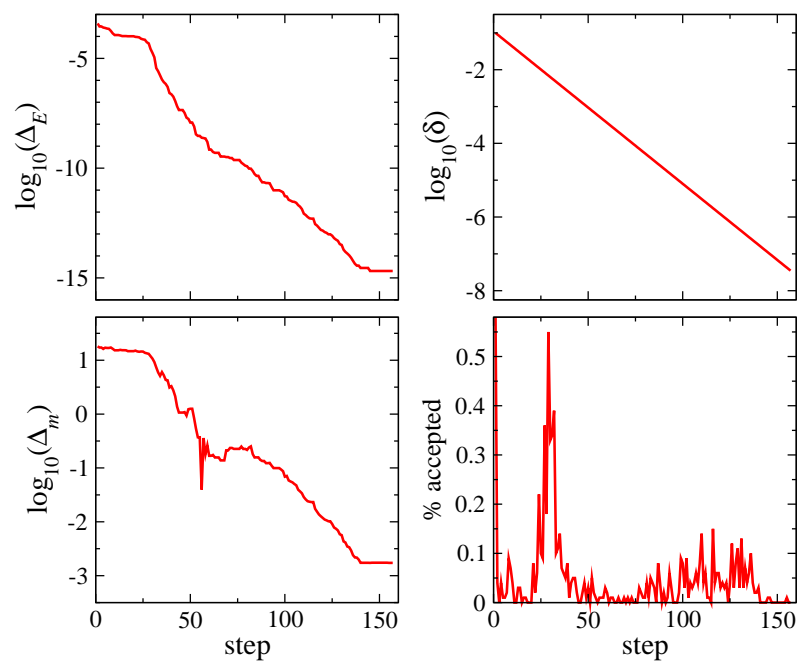

FIG. 2: (Color online) Stochastic energy minimization with $10^{4}$ updates per step for a $D=4$ MPS at $h / J=1.01432$, using 64-bit floating point arithmetic. The relative energy and magnetization errors are defined as $\Delta_{E}=\left(E-E_{\infty}\right) / E_{\infty}$, $\Delta_{m}=|| m|-| m_{\infty}|| /\left|m_{\infty}\right|$, where the subscript $\infty$ refers to results converged at the 128 -bit level.

most efficient approach is Vidal's time evolving block decimation (TEBD) scheme [10], where the ground state is projected out in the limit of long imaginary time [3, 11], starting from an initial (e.g., random) state. Similar methods have also been developed for two-dimensional iPEPSs, where expectation values cannot simply be expressed in eigenvalue forms such as (6), but where good approximations to the contractions can still be defined and evaluated using TEBD-like methods [16].

Here we investigate symmetry breaking and critical scaling of the order parameter in the transverse-field Ising model. In one dimension the hamiltonian is

$$
H=-J \sum_{i=1}^{N} \sigma_{i}^{z} \sigma_{i+1}^{z}-h \sum_{i=1}^{N} \sigma_{i}^{x},
$$

with periodic boundary conditions. This model is exactly solvable 21] and has a paramagnetic-magnetic $(m \neq 0)$ transition at $h_{c} / J=1$. In two dimension, the critical point has been determined using quantum Monte Carlo calculations, giving $h_{c} / J \approx 3.044$ [22]. Single-spin meanfield theory $(D=1, N=\infty)$ gives $h_{c} / J=2$ and 4 in one and two dimensions, respectively, and the mean-field form of the magnetization is $m \sim\left(h_{c}-h\right)^{\beta}$ for $h<h_{c}$, with $\beta=1 / 2$. The exact critical exponent is $\beta=1 / 8$ in one dimension and $\beta \approx 0.325$ in two dimensions.

Considering first MPSs, we optimize the $A$ matrices (which we take as real and symmetric) using two different stochastic schemes; one using derivatives and one using only the energy. While the convergence is very slow for large $D$ compared to state-of-the-art TEBD [11], the methods do not rely on any approximations and are numerically stable. With stochastic updates, we can avoid potential local minimums in a complex energy landscape. 


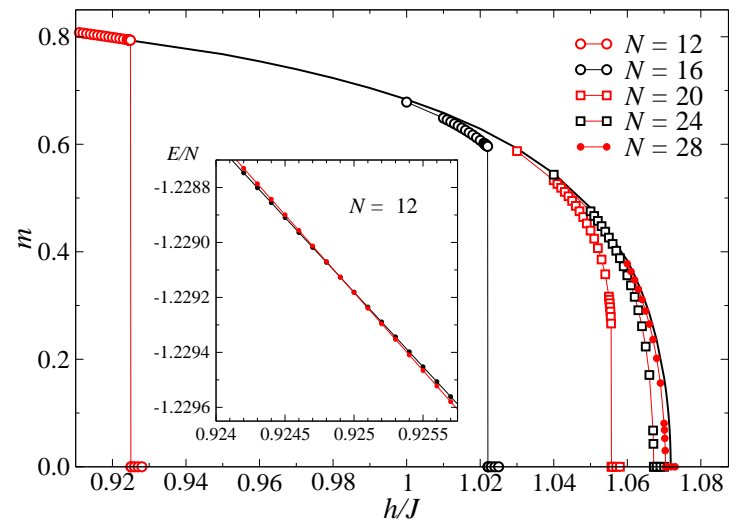

FIG. 3: (Color online) Magnetization curves for $D=2$ and different system sizes. The solid curve is for $N=\infty$. The inset shows two almost degenerate energy minimums for $N=$ 12 , which cross each other at the transition.

The derivative-based method is the one used in 24], but with exactly computed energies and derivatives for finite $N$. For $N=\infty$, we instead use a brute-force scheme with completely random simultaneous updates of all the matrix elements (but keeping the matrices symmetric); $A_{a b}(\sigma) \rightarrow A_{a b}(\sigma)+\delta\left[1 / 2-r_{a b}(\sigma)\right]$, with uniformly distributed random numbers $r_{a b}(\sigma) \in[0,1)$. An update is accepted only if the energy decreases, and then the matrices are normalized so that the largest $\left|A_{a b}(\sigma)\right|=1$. One step of this procedure typically involves $n \sim 10^{3}-10^{4}$ trials. If the acceptance rate is below $10 \%$ we reduce $\delta$ by dividing by, e.g., 1.1. To ensure full convergence, when $\delta$ has reached the limit where the updates no longer can influence the energy (within the numerical precision), it is reset to a larger value and the process is repeated (several times, until no updates are accepted).

Fig. 2 illustrates the brute-force procedure for a $D=4$ MPS optimized at $h / J=1.01432$. The evolution of the errors of the energy and the magnetization is shown, along with $\delta$ and the acceptance rate. In this case the acceptance rate was always below $10 \%$, and $\delta$ therefore decreases after each step. This calculation was carried out using standard 64-bit floating-point arithmetic, which is reflected in the convergence of the energy to within a relative error of $\approx 10^{-15}$. The computation was continued with 128-bit arithmetic, until the energy was converged to $\approx 10^{-25}$. The errors graphed in the figure are with respect to this second optimization. The 64-bit optimization took only a few minutes, whereas the subsequent 128-bit run took many hours. The computational effort increases very rapidly with $D$, and we have only carried out systematic studies up to $D=10$ (for which some points required several weeks of CPU time) [23].

Note that while the energy in Fig. 2 has converged to full 64-bit precision, the relative magnetization error is much larger, $\Delta_{m} \approx 10^{-3}$. Using 128-bit arithmetic gives $m=0.031814167$ (where all digits shown are converged). It is well known that the energy in MPS and DMRG cal-

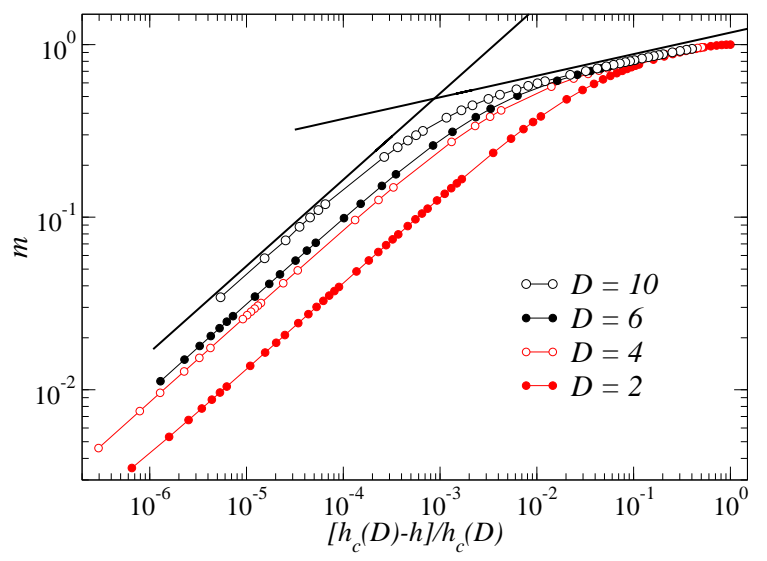

FIG. 4: (Color online) Demonstration of asymptotic MPS mean-field behavior and scaling cross-over in one dimension. The $D$-dependent critical fields are: $h_{c} / J=1.0717967(D=$ $2), 1.0143343(D=4), 1.0063523(D=6) 1.0021654(D=$ 10). The lines have slopes $\beta=1 / 8$ and $1 / 2$.

culations converges much faster than other quantities [5], but $N=\infty$ results close to the critical point appear to be even more sensitive to extremely small energy variations than had been previously anticipated. When trying to extract the asymptotic critical behavior of $m$, the problem is accentuated by the fact that it is the relative, not absolute error that is relevant. All results to be discussed below have been converged to the level required for a reliable scaling analysis.

As shown in Fig. 3, for finite $N$ the phase transition occurs with sharp magnetization jumps for small $N$, which become less pronounced as $N$ increases and the transition moves toward higher $h$. The curves converge toward the continuous transition obtained in the infinite- $N$ calculation. The first-order behavior can be traced to the presence of two energy minimums (shown in Fig. 3 for $N=12$ ), which we can track using steepest-decent optimizations starting from large and small $h$ (changing $h$ slowly). The diminishing discontinuity with increasing $N$ implies that the minimums move closer to each other in parameter space, coinciding at $h_{c}$ for $N=\infty$. For fixed finite $N$, the discontinuous jumps move toward $h=0$ with increasing $D$, reflecting the fact that when $D \rightarrow \infty$ an MPS can reproduce the exact spin-inversion symmetric $(m=0)$ ground state of a finite chain.

For $N=\infty$ and any $D$, the optimal state is symmetrybroken below some $h_{c}(D)$, with $h_{c}(D) / J \rightarrow 1$ as $D \rightarrow \infty$. The $D$ dependence is not smooth, as has been pointed out before [15]. Here we focus on the behavior of $m$ for $h \rightarrow h_{c}(D)$. Thanks to our high-precision data, we can extract $h_{c}(D)$ reliably using a power-law assumption; $m \propto\left(h_{c}-h\right)^{\beta}$ for $0<m \ll 1$. This always gives $\beta \approx$ 0.50 for the best fit, suggesting that the MPS procedure leads to mean-field behavior for any finite $D$. As shown in Fig. 4, the true critical behavior $(\beta=1 / 8)$ emerges within a window of $h$-values with increasing $D$, with the cross-over to $\beta=1 / 2$ gradually moving toward $h_{c}$. 


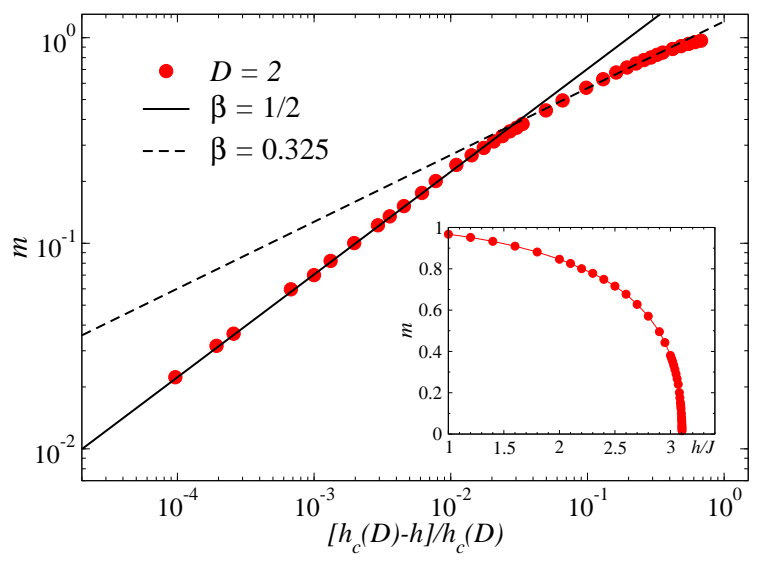

FIG. 5: (Color online) Field dependence of the magnetization computed with a $D=2$ iPEPS in two dimensions. The critical field is $h_{c} / J=3.1041$.

It is perhaps not surprising, after all, that a finite$D$ MPS cannot reproduce a non-trivial critical exponent asymptotically, because the correlation length is finite. Criticality (which can be non-mean-field) in a onedimensional classical Ising model requires long-range interactions [25] and the partition function then does not correspond to an MPS with finite $D$. It has also been proved that a finite- $D$ MPS can be renormalized to a product state [26]. It is, however, remarkable that the system is so sensitive to incomplete optimization that the asymptotic mean-field behavior of the order parameter had not been noted in previous studies [15, 18].

We now turn to the two dimensional iPEPS. Nontrivial criticality has been anticipated in this case, even for finite $D$ [7], because partition functions of classical models with critical points can be written as tensor products [13]. Magnetization curves closely following the expected power-law with $\beta \approx 0.325$ have been reported [16, 17, 19], but the calculations are not very accurate close to the critical point. We have used an improved iPEPS contraction scheme [20], which is less affected by approximations. Fig. 5 shows transverse-field Ising results for $D=2$. An asymptotic mean-field behavior is seen unambiguously, and further away from the critical point there is again a cross-over to a behavior matching closer the true $\beta$. However, for $D=2$ the cross-over takes place where $m$ is already large, $\approx 0.5$, and this is not actual critical behavior. Unless $D$ is much larger, there will be no clear-cut scaling with the correct exponent.

Our study shows that great care has to be taken when extracting critical scaling forms from the order parameter in MPS and PEPS calculations. Asymptotic meanfield behavior should be expected, not just for the Ising models considered here, but at symmetry-breaking transitions in general. This information helps to accurately locate the critical point for small $D$. To extract the true critical behavior, it is necessary to carefully examine the behavior for increasing $D$.
Acknowledgments-We would like to thank P.-C. Chen, I. McCulloch, D. Perez-Garcia, and F. Verstraete for useful discussions, and I. McCulloch also for providing TEBD results for comparisons 23]. AWS is supported by NSF grant No. DMR-0803510 and would also like to thank the NCTS of Taiwan for hospitality and funding during a visit. YJK is supported by NCTS and NSC of Taiwan under grants NSC 97-2628-M-002-011-MY3 and NTU 98R0066-65, -68.

[1] I Affleck, T. Kennedy, E. Lieb, and H. Tasaki, Commun. Math. Phys. 115, 477 (1988).

[2] S. Östlund and S. Rommer, Phys. Rev. Lett. 75, 3537 (1995).

[3] F. Verstraete, V. Murg, and J. I. Cirac, Adv. Phys. 57, 143, (2008).

[4] S. R. White, Phys. Rev. Lett. 69, 2863 (1992).

[5] U. Schollwöck, Rev. Mod. Phys. 77, 259 (2005).

[6] F. Verstraete and J. I. Cirac, Phys. Rev. B 73, 094423 (2006).

[7] F. Verstraete, M. M. Wolf, D. Perez-Garcia, and J. I. Cirac, Phys. Rev. Lett. 96, 220601 (2006).

[8] M. B. Hastings, Phys. Rev. B 76, 035114 (2007).

[9] V. Murg, F. Verstraete, and J. I. Cirac, Phys. Rev. A 75, 033605 (2007).

[10] G. Vidal, Phys. Rev. Lett. 98, 070201 (2007).

[11] I. P. McCulloch, arXiv:0804.2509.

[12] G. Vidal, Phys. Rev. Lett. 93, 040502 (2004).

[13] T. Nishino, K. Okunishi, Y. Hieida, N. Maeshima, and Y. Akutsu, Nucl. Phys. B 575, 504 (2000).

[14] F. Verstraete and J. I. Cirac, Arxiv:cond-mat/0407066.

[15] L. Tagliacozzo, T. R. de Oliveira, S. Iblisdir, and J. I. Latorre, Phys. Rev. B 78, 024410 (2008).

[16] J. Jordan, R. Orús, G. Vidal, F. Verstraete, and J. I. Cirac, Phys. Rev. Lett. 101, 250602 (2008).

[17] R. Orús and G. Vidal, Phys. Rev. B 80, 094403 (2009).

[18] D. Nagaj, E. Farhi, J. Goldstone, P. Shor, and I. Sylvester, Phys. Rev. B 77, 214431 (2008).

[19] Z.-C. Gu, M. Levin, and X.-G. Wen, Phys. Rev. B 78, 205116 (2008).

[20] L. Wang and F. Verstraete (unpublished).

[21] T. D. Shultz, D. C. Mattis and E. H. Lieb, Rev. Mod. Phys. 36, 856 (1964).

[22] H. Rieger and N. Kawashima, Eur. Phys. J. B 9, 233 (1999).

[23] We have compared 64-bit $D=4$ results with TEBD calculations (also 64-bit) (I. McCulloch, private communication). The energies agree perfectly to 15 -digit precision, whereas the TEBD magnetization is typically somewhat closer to the final result of the 128-bit stochastic calculation. Since $m$ can fluctuate by some amount $\Delta_{m}$ without changing the energy at a given level of precision, the result within $\pm \Delta_{m}$ depends on details of the optimization method and the starting state. Even for $D$ as small as 4, the TEBD calculation also converges very slowly to full 64-bit precision (taking several days close to $h_{c}$ ).

[24] A. W. Sandvik and G. Vidal, Phys. Rev. Lett. 99, 220602 (2007).

[25] P. W. Anderson and G. Yuval, J. Phys. C 4, 607 (1971).

[26] F. Verstraete, J. I. Cirac, J. I. Latorre, E. Rico, and M. M. Wolf, Phys. Rev. Lett. 94140601 (2005). 